\title{
Aggregation-Free DNA Nanocage/Quantum Dot Complexes based on Electrostatic Adsorption
}

\author{
Zhenguang Wang ${ }^{\mathrm{a}}$, Tam Dick Yan ${ }^{\mathrm{b}}$, Andrei S. Susha ${ }^{\mathrm{a}}$, Miu Shan Chan ${ }^{\mathrm{b}}$, Stephen V. \\ Kershaw $^{\mathrm{a}}$, Pik Kwan Lo ${ }^{\mathrm{b}}$, Andrey L. Rogach ${ }^{\mathrm{a} 1}$ \\ ${ }^{a}$ Department of Physics and Materials Science and Centre for Functional Photonics (CFP), City University of Hong Kong, 83 Tat \\ Chee Avenue, Kowloon, Hong Kong SAR \\ ${ }^{\mathrm{b}}$ Department of Biology and Chemistry, City University of Hong Kong, Kowloon, 83 Tat Chee Avenue, Kowloon, Hong Kong \\ SAR \\ ${ }^{1}$ Corresponding author. E-mail: andrey.rogach@ cityu.edu.hk
}

\begin{abstract}
Fabrication of DNA/Quantum Dot (QD) complexes making use of electrostatic adsorption forces has advantages of the cost-efficiency and simplicity. To obtain aggregation-free complexes, high molecular weight polymers or the purposefully designed ligands are commonly employed as a bridge between DNA and QDs, which limits their applications in practice. In this work, DNA/QDs complexes were obtained using electrostatic adsorption between cysteamine stabilized CdTe QDs and self-assembled DNA nanocages. Zeta potential, dynamic light scattering and gel electrophoresis measurements demonstrated their aggregation-free state, different from the cases where single-stranded and double-stranded DNAs were used. The appearance of aggregation-free complexes in the case of DNA nanocages was ascribed to their 3D rigid structure. Förster resonance energy transfer (FRET) was demonstrated for the complexes of Cy3 labelled DNA nanocages and QDs, pointing out on the close proximity of these building blocks.
\end{abstract}

\section{Keywords}

Quantum Dots; DNA/QDs interaction; DNA nanocages; FRET 


\section{Introduction}

Owing to their molecular recognition properties and the nucleobase sequence controllability, DNAs are emerging as powerful and versatile building blocks for the construction of nanostructures $[1,2]$. Both two- and three-dimensional DNA nanostructures can be precisely designed [2-7]. An elegant strategy was developed to assemble DNA strands into well-defined DNA nanocage structures, with a high yield and stability [8-10]. Much attention has been focused on construction of DNA/quantum dots (QDs) complexes [11-14]. Such complexes combine the unique optical properties of QDs with the molecular recognition ability of DNA, which has broad implications in the fields of material science [15-18] and biosensing [19-21]. The most important step in the construction of DNA/QDs complexes is the attachment of DNA to the QD surface. Depending on the surface characteristics of QDs and DNA, several attachment methods have been developed, including streptavidin-biotin linking methods [22, 23], covalent bonding methods using $-\mathrm{COOH},-\mathrm{NH}_{2}$ or $-\mathrm{SH}$ groups [24-26], and electrostatic adsorption methods [20, 27, 28]. DNA based nanostructures have a negative charge in solutions with neutral $\mathrm{pH}$. In order to construct DNA/QDs complexes making use of electrostatic adsorption forces, QDs should be either positively charged by employing appropriate ligands at the synthesis stage, or a positively charged layer has to be applied onto QD surface postpreparatively. During the electrostatic adsorption process, the positive charge of the QDs will be (partially) neutralized by the negative charge of the DNA, which may adversely influence their colloidal stability [14]. To prevent QDs from aggregation, positively charged polymers were often employed as a bridge between QDs and DNA [20, 28, 29]. However, polymers have high molecular weight, leading to a large distance between DNA and QDs. Several groups used positively charged ligands on the QDs for the construction of DNA/QDs complexes [30, 31]. Lee et al designed positively charged QDs using DHLA-2,2'-(ethylenedioxy)bis(ethylamine) trifluoroacetate [30], while Rotello's group developed a two-step ligand exchange process [32]. Such approaches require complicated ligand synthesis and design, which limits their applications in practice. Therefore, it is highly desirable to fabricate aggregation-free DNA/QDs complexes using electrostatic adsorption, while avoiding large molecular weight polymers and complicated ligand design.

In this work, DNA/QD complexes were obtained by using electrostatic adsorption between positively charged cysteamine stabilized CdTe QDs and self-assembled DNA nanocages. Zeta potential measurements, dynamic light scattering, gel electrophoresis, and optical spectroscopy were applied to confirm the aggregation-free nature of the DNA nanocage/QD complexes, which was different for the cases when single-stranded DNAs (ssDNA) and double stranded DNAs (dsDNA) were employed. Förster resonance energy transfer (FRET) was demonstrated for the complex of Cy3 labeled DNA nanocages and QDs, pointing out on the close proximity of these building blocks. 


\section{Materials and methods}

\subsection{Materials}

All chemicals including cadmium acetate di-hydrate, tellurium powder, sodium borohydride, cysteamine hydrochloride, acetic acid, N,N',N'-tetramethylethylenediamine, glycerol, and acrylamide were purchased from Sigma-Aldrich. N,N'-Methylenebis (acrylamide), urea, boric acid, formamide, magnesium chloride, EDTA, tris (hydroxymethyl) aminomethane (Tris), (3aminopropyl) trimethoxysilane, ammonium persulfate were purchased from J\&K. Agarose was purchased from Vivantis. HydraGreen ${ }^{\mathrm{TM}}$ Safe DNA Dye was purchased from ACTGene. DNA sequences were purchased from IDT. TAMg buffer $(1 \mathrm{X})$ is consisted of $45 \mathrm{mM}$ Tris, $7.6 \mathrm{mM}$ $\mathrm{MgCl}_{2}$, with $\mathrm{pH}$ adjusted to 7.8 using glacial acid. PBS Buffer $(1 \mathrm{X})$ is consisted of $137 \mathrm{mM}$ $\mathrm{NaCl}, 2.7 \mathrm{mM} \mathrm{KCl}, 10 \mathrm{mM} \mathrm{Na} \mathrm{HPO}_{4}, 2 \mathrm{mM} \mathrm{KH} \mathrm{PO}_{4}$, with $\mathrm{pH}$ adjusted to $7.4 \mathrm{msing} \mathrm{HCl}$. TA buffer $(1 \mathrm{X})$ is consisted of $40 \mathrm{mM}$ Tris with $\mathrm{pH}$ adjusted to $\mathrm{pH} 8.0 \mathrm{using}$ Glacial acetic acid. All other chemicals were used as received. The DNA sequences and the related information are presented in Table S1.

\subsection{Assembly of DNA nanocages and Cy3 labeled DNA nanocages}

The methods to assemble DNA nanocages and Cy3 labeled DNA nanocages together with their characterization data are presented in SI.

\subsection{Synthesis of cysteamine stabilized CdTe QDs}

Cysteamine stabilized CdTe QDs were prepared according to the previous report [33]. The concentration of QDs was determined according to the ref. [34]. The emission quantum yield of QDs was in the range of 5 to $18 \%$, estimated using Rhodamine 6G in ethanol as a standard.

\subsection{Assembly of DNA/QDs complexes}

Before use, cysteamine stabilized QDs were precipitated by 2-propanol, and re-dissolved in a DI-water to achieve the concentration of $2.5 \mu \mathrm{M}$. Typically, $5.4 \times 10^{-11} \mathrm{~mol}$ of ssDNA or dsDNA was mixed with $1.8 \times 10^{-11}$ mol of QDs, respectively. The ratio of ssDNA and dsDNA to QDs was 3:1. For assembly of DNA nanocage/QD complexes, $1.8 \times 10^{-11}$ mol of DNA nanocages was mixed with $1.8 \times 10^{-11} \mathrm{~mol}$ of QDs. The ratio of DNA nanocages to QDs was 1:1. DI water was used to adjust the volume into $80 \mu \mathrm{L}$. All the measurements were conducted after incubation of solutions for 30 min under room temperature.

\subsection{Characterization}

Absorption and photoluminescence (PL) spectra of samples were measured directly on Varian Cary 50 UV-visible spectrophotometer and Cary Eclipse fluorescence spectrometer, respectively. The hydrodynamic diameter (number-weighted mean diameter) and zeta potentials of QDs and DNA/QDs complexes were measured by dynamic light scattering (DLS) on a Zeta 
Sizer Nano. Before measurements, the ssDNA/QDs, dsDNA/QDs and DNA nanocage/QD complexes were mixed with $1 \mathrm{~mL}$ of PBS buffer $(1 \mathrm{X})$. The final concentrations of QDs, ssDNA, dsDNA and DNA nanocages were $18 \mathrm{nM}, 54 \mathrm{nM}, 54 \mathrm{nM}$ and $18 \mathrm{nM}$, respectively. UV/vis measurements of DNA were conducted on NanoDrop spectrophotometer (ND-1000). Gel electrophoresis experiments regarding native or denaturing gel were carried out on an acrylamide 20 X $20 \mathrm{~cm}$ Maxi Vertical electrophoresis apparatus (MV-20DSYS), in TAMg buffer (1 X). Agarose gel electrophoresis was conducted on Midi plus-2 Horizontal Electrophoresis System (ME15-7-10-15), in TA buffer (1 X). Gel scanning was performed by Fujifilm ImageQuant LAS 4000 .

\section{Results and discussion}

\subsection{Construction of DNA nanocage/QD complexes}

Cysteamine stabilized CdTe QDs synthesized at a neutral $\mathrm{pH}$ show a positive charge (zetapotential: $+32.6 \mathrm{mV}$ ), which makes them suitable for fabrication of DNA/QDs complexes. They possess a continuous broad absorption stretching from 400 to $600 \mathrm{~nm}$ and a PL emission maximum at $625 \mathrm{~nm}$.

Scheme 1 illustrates 3 different types of DNA/QDs complexes created by electrostatic adsorption between cysteamine stabilized QDs and DNA nanocages, ssDNA and dsDNA. Zeta potential changed from $+32.6 \mathrm{mV}$ for the bare QDs to $-32.9 \mathrm{mV}$ for the DNA nanocage/QD complex. For the ssDNA and dsDNA, zeta potential changed to -37.8 and $-39.3 \mathrm{mV}$, respectively.

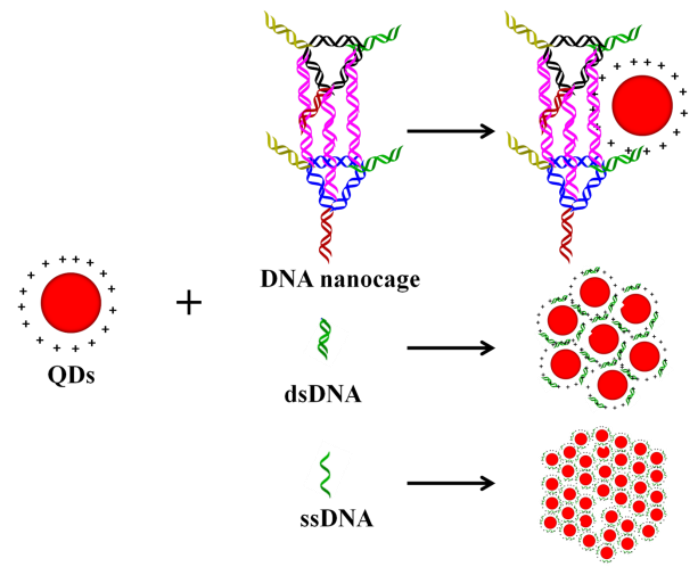

Scheme 1. Schematic illustration of 3 different kinds of DNA/QDs complexes, depending on the structure of the DNA building blocks used.

3.2. Dynamic light scattering 
The hydrodynamic diameter of DNA/QDs complexes is a critical parameter allowing us to estimate their aggregation degree in solution. A common standard for the aggregation-free bare QDs is that their hydrodynamic diameter should be less than double of the TEM-estimated core size [35, 36]. As shown in Figure 1a, the hydrodynamic diameter of bare QDs and the DNA nanocage/QD complex is 6.5 and $16.9 \mathrm{~nm}$, respectively. Estimated from the DLS, the hydrodynamic diameter of the bare DNA nanocages is about $7.6 \mathrm{~nm}$ [10]. The smallest DNA nanocage/QDs complex is one DNA nanocage coupled with one QD and the hydrodynamic diameter of such a complex can be estimated as $\sim 14 \mathrm{~nm}$. From the experimental data of Figure 1a, we conclude that the DNA nanocage/QD complex forms preferably in such a monomeric state. In comparison, the hydrodynamic diameter of ssDNA/QD and dsDNA/QDs complexes were estimated as $122 \mathrm{~nm}$ and $28 \mathrm{~nm}$, respectively, being much larger. Even so the aggregation of the dsDNA/QD complex appears to be less severe than for ssDNA/QD complex, both of them are strongly affected by agglomeration.

\subsection{Agarose gel electrophoresis}

Aggregation states of DNA/QD complexes were further studied by the agarose gel electrophoresis. As shown in Figure 1b, two DNA bands with lower mobility and reduced DNA band intensity of free ssDNA or dsDNA were observed after adding the QD to the ssDNA or dsDNA (lane 4 and 5), as compared with only ssDNA or dsDNA (lane 1 and 2). This indicated that the aggregation took place and some higher molecular weight products/complexes were formed. In contrast, a slightly slower mobility DNA band is observed and no high molecular weight product is formed after adding QDs to the self-assembled DNA nanocages (lane 6) as compared to the free DNA nanocages (lane 3). These results revealed an absence of aggregation in the latter case, being consistent with observations related to hydrodynamic diameters. 


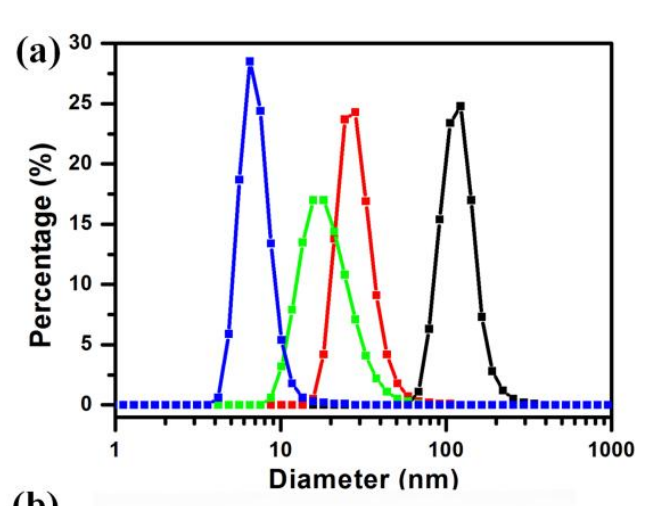

(b)

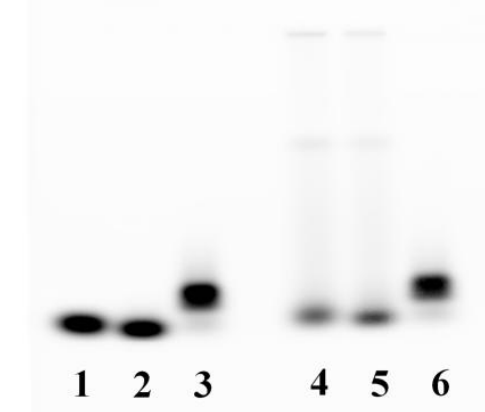

Fig. 1. (a) Size distribution of bare QDs (blue line), ssDNA/QDs complex (black line), dsDNA/QDs complex (red line) and DNA nanocage/QD complex (green line). (b) 3\% agarose gel electrophoresis of ssDNA (line 1), dsDNA (line 2), DNA nanocages (line 3), ssDNA/QDs complex (line 4), dsDNA/QDs complex (line 5), and DNA nanocage/QD complex (line 6).

The difference of the aggregation degree among DNA nanocage/QD, ssDNA/QD and dsDNA/QD complexes most probably originates from the different rigidity of these three DNA structures. ssDNA is flexible and dsDNA is more rigid, while the DNA nanocages have most rigid 3D structure. The formation of aggregation-free DNA nanocage/QD complexes is thus attributed to the 3D rigid structure of the DNA nanocages, which different to dsDNA and in particulate to ssDNA provides a strong steric repulsion preventing the complexes formed from aggregation (see Scheme 1 for illustration).

\subsection{Optical spectroscopy and FRET study}

FRET is a classical tool to study molecular conformational changes and intermolecular distances between chromophores [37-40]. A typical FRET system consists of a donor and an acceptor. The energy of a donor, initially in its electronic excited state, transfers to an acceptor through nonradiative dipole-dipole coupling, when the distance between donor and acceptor is small enough, as determined by the Förster radius. In order to confirm the close proximity of 
chromophores within the DNA nanocage/QD complex, FRET process between Cy3 dye (donor) labeled DNA nanocages and QDs (acceptor) was studied. Each DNA nanocage was labeled by three Cy3 dye molecules, and after mixing Cy3 labeled DNA nanocages with QDs, Cy3 DNA nanocage/QD complexes have been constructed. Owing to the continuous and broad absorption of QDs (from 400 to $600 \mathrm{~nm}$ ), there is an overlap between the absorption of QDs and the emission of Cy3 dye (maximum at $564 \mathrm{~nm}$ ). Thus, a FRET pair is formed with a Cy3 as a donor and QDs as acceptor. The absorption spectra of the equal concentrations of QDs, Cy3 DNA nanocages and of Cy3 DNA nanocage/QD complex are shown in Figure 2a. For wavelengths above $580 \mathrm{~nm}$, the absorption of Cy3 is negligible, whereas the absorption spectrum of QDs is almost the same as that of the Cy3 DNA nanocage/QD complex. This demonstrates that the band gap of QDs is not affected during the formation of DNA nanocages/QDs complex. The occurrence of FRET is obvious in the PL spectra excited at the wavelength of $540 \mathrm{~nm}$, as shown in Figure 2b. Upon formation of the Cy3 DNA nanocage/QD complex, the PL intensity of Cy3 on DNA nanocages is quenched by 3.6-fold and the PL intensity of QDs is almost doubled. Noticing that, there is a slight red shift on the PL spectrum after forming Cy3 DNA nanocage/QD complex, which may be caused by the change of zeta potentials. This demonstrates the close proximity between the DNA nanocage and the QDs, and further confirms the formation of the DNA nanocage/QD complex.
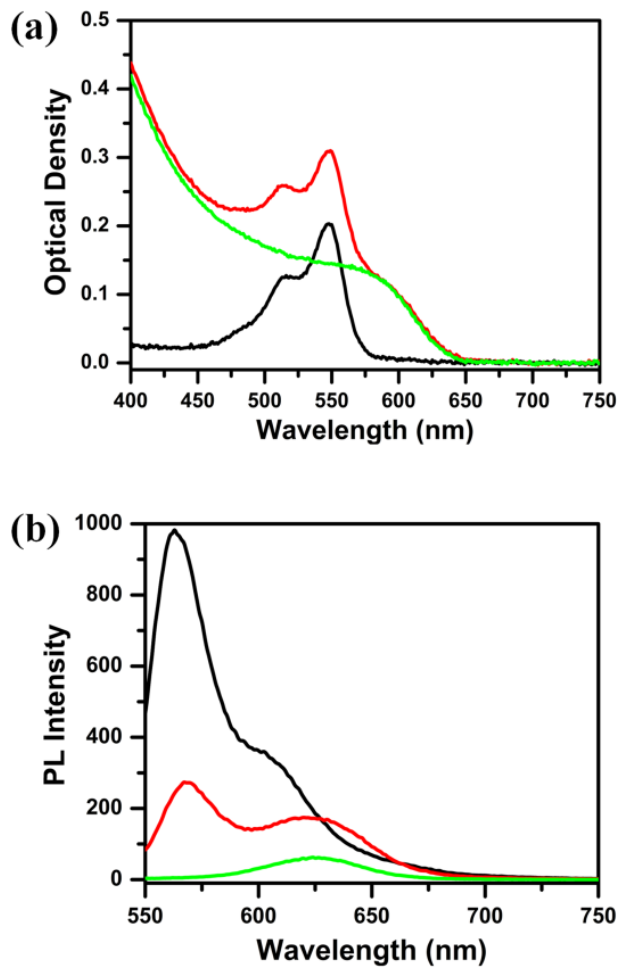
Fig. 2. (a) Absorption and (b) emission spectra of QDs (green line), Cy3 labeled DNA nanocages (black line) and Cy3 DNA nanocage/QD complex (red line). All samples were excited at $540 \mathrm{~nm}$, the optimal excitation wavelength for Cy3.

The FRET efficiency can be calculated based on the absorption and emission spectra. However, in our case there is an overlap between the emission spectra of Cy3 and QDs, which is hard to separate from. In order to calculate the FRET efficiency, the spectra of QDs and Cy3 in DNA cage have been fitted to Gaussian functions (as shown in Figure S5). Above Gaussians are further used to fit PL spectrum of Cy3 DNA nanocage/QD complex. After adding a red shift parameter $(20 \mathrm{meV})$, a good fitting to the PL spectrum of Cy3 DNA nanocage/QD complex was obtained, as shown in Figure 3. Fitting formulae

$\mathrm{F}_{\text {complex }}(\mathrm{E})=0.269 \times \mathrm{F}_{\mathrm{Cy} 3}(\mathrm{E})+1.886 \times \mathrm{F}_{\mathrm{QDs}}(\mathrm{E})$

can be used, where $\mathrm{F}_{\text {complex }}(\mathrm{E}), \mathrm{F}_{\mathrm{Cy} 3}(\mathrm{E})$ and $\mathrm{F}_{\mathrm{QDs}}(\mathrm{E})$ are the best fitting functions for the PL spectra of the Cy3 DNA nanocage/QD complex, Cy3 DNA nanocage and QDs, respectively. The FRET efficiency can be calculated as $E=1-\left(\Phi_{\mathrm{D}-\mathrm{A}} / \Phi_{\mathrm{D}}\right)$, where $\Phi_{\mathrm{D}-\mathrm{A}}$ and $\Phi_{\mathrm{D}}$ are the quantum efficiencies of the Cy3 DNA nanocage/QD complex and the Cy3 DNA nanocage [41]. In our case, it can be rewritten as $E=1-0.269 \times \mathrm{A}_{\mathrm{D}} / \mathrm{A}_{\mathrm{D}-\mathrm{A}}$, where are $\mathrm{A}_{\mathrm{D}-\mathrm{A}}$ and $\mathrm{A}_{\mathrm{D}}$ are the absorption of Cy3 DNA nanocage/QD complex and Cy3 DNA nanocage at the excitation wavelength (540 $\mathrm{nm})$. Applying above equation to the absorption spectra, $E$ equals to $84 \%$.

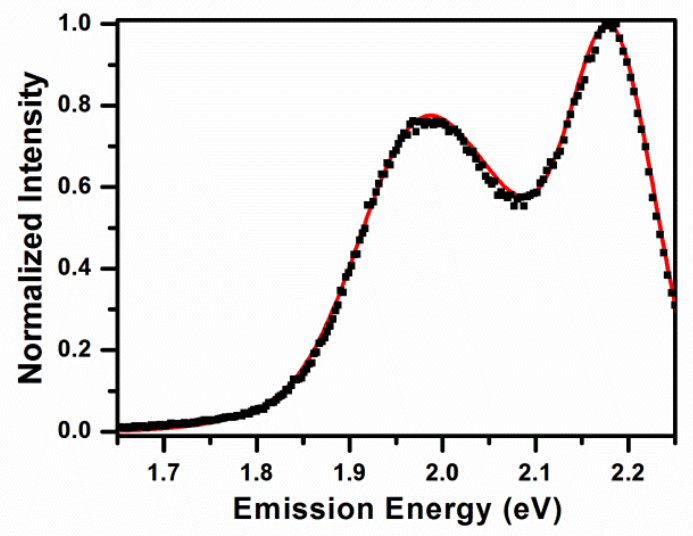

Fig. 3. PL spectrum of the Cy3 DNA nanocage/QD complex (dotted line) estimated by a fitting function (1), solid red line.

\section{Conclusions}

In summary, aggregation-free DNA nanocage/QD complexes have been constructed using electrostatic adsorption between positively charged, cysteamine stabilized CdTe QDs and negatively charged, self-assembled DNA nanocages, without the need for molecular binders such 
as polymers or specially designed QD ligands. The formation of DNA nanocage/QD complexes was confirmed by zeta potential measurements, DLS and gel electrophoresis, which also points out that the complexes obtained is in an individual monomeric state. ssDNAs and dsDNAs were also able to form complexes with QDs, but with a high degree of aggregation, which is related to the different rigidity of these three DNA structures. The occurrence of FRET, with a efficiency of $84 \%$, in a system of Cy3-labeled DNA nanocage/QD complexes further confirms the close proximity between the DNA and QDs. Stable, aggregation-free DNA nanocage/QD complexes presented here may found applications in biosensing.

\section{Acknowledgements}

This work has been supported by the Centre for Functional Photonics, City University of Hong Kong, by the National Science Foundation of China (21324077) and Hong Kong Research Grants Council (21300314).

\section{References}

[1] N.C. Seeman, Nucleic acid junctions and lattices, J. Thero. Biol. 99 (1982) 237-247.

[2] F.A. Aldaye, A.L. Palmer, H.F. Sleiman, Assembling materials with DNA as the guide, Science 321 (2008) 1795-1799.

[3] T.J. Fu, N.C. Seeman, DNA double-crossover molecules, Biochemistry 32 (1993) 3211-3220.

[4] P.W. Rothemund, Folding DNA to create nanoscale shapes and patterns, Nature 440 (2006) 297-302.

[5] A.V. Pinheiro, D. Han, W.M. Shih, H. Yan, Challenges and opportunities for structural DNA nanotechnology, Nature Nanotech. 6 (2011) 763-772.

[6] Z. Dai, D.Y. Tam, H. Xu, M.S. Chan, L.S. Liu, F. Bolze, X.H. Sun, P.K. Lo, Conformational change of self-aAssembled DNA nanotubes induced by two-photon excitation, Small (2015) doi: 10.1002/smll.201500333.

[7] D.Y. Tam, P.K. Lo, Multifunctional DNA nanomaterials for biomedical applications, J Nanomater, (2015) doi:10.1155/2015/765492.

[8] F.A. Aldaye, H.F. Sleiman, Modular access to structurally switchable 3D discrete DNA assemblies, J. Am. Chem. Soc. 129 (2007) 13376-13377.

[9] C.K. McLaughlin, G.D. Hamblin, F.A. Aldaye, H. Yang, H.F. Sleiman, A facile, modular and high yield method to assemble three-dimensional DNA structures, Chem. Commun. 47 (2011) 8925-8927.

[10] M.S. Chan, P.K. Lo, Nanoneedle-Assisted Delivery of Site-Selective PeptideFunctionalized DNA Nanocages for Targeting Mitochondria and Nuclei, Small 10 (2014) 12551260.

[11] C.Y. Zhang, H.C. Yeh, M.T. Kuroki, T.H. Wang, Single-quantum-dot-based DNA nanosensor, Nat. Mater. 4 (2005) 826-831.

[12] C.Y. Zhang, J. Hu, Single quantum dot-based nanosensor for multiple DNA detection, Anal. Chem. 82 (2010) 1921-1927.

[13] H. Wang, R. Yang, L. Yang, W. Tan, Nucleic acid conjugated nanomaterials for enhanced molecular recognition, ACS Nano 3 (2009) 2451-2460. 
[14] D. Sun, O. Gang, DNA-functionalized quantum dots: fabrication, structural, and physicochemical properties, Langmuir 29 (2013) 7038-7046.

[15] A. Samanta, Z. Deng, Y. Liu, Infrared emitting quantum dots: DNA conjugation and DNA origami directed self-assembly, Nanoscale 6 (2014) 4486-4490.

[16] A. Samanta, S. Banerjee, Y. Liu, DNA nanotechnology for nanophotonic applications, Nanoscale 7 (2015) 2210-2220.

[17] Z. Deng, A. Samanta, J. Nangreave, H. Yan, Y. Liu, Robust DNA-functionalized core/shell quantum dots with fluorescent emission spanning from UV-vis to near-IR and compatible with DNA-directed self-assembly, J. Am. Chem. Soc. 134 (2012) 17424-17427.

[18] R. Schreiber, J. Do, E.M. Roller, T. Zhang, V.J. Schuller, P.C. Nickels, J. Feldmann, T. Liedl, Hierarchical assembly of metal nanoparticles, quantum dots and organic dyes using DNA origami scaffolds, Nature Nanotech. 9 (2014) 74-78.

[19] S. Su, J. Fan, B. Xue, L. Yuwen, X. Liu, D. Pan, C. Fan, L. Wang, DNA-conjugated quantum dot nanoprobe for high-sensitivity fluorescent detection of DNA and micro-RNA, ACS Appl. Mater. Interfaces 6 (2014) 1152-1157.

[20] H. Peng, L. Zhang, T.H. Kjallman, C. Soeller, J. Travas-Sejdic, DNA hybridization detection with blue luminescent quantum dots and dye-labeled single-stranded DNA, J. Am. Chem. Soc. 129 (2007) 3048-3049.

[21] R. Freeman, J. Girsh, I. Willner, Nucleic acid/quantum dots (QDs) hybrid systems for optical and photoelectrochemical sensing, ACS Appl. Mater. Interfaces 5 (2013) 2815-2834.

[22] I.L. Medintz, H.T. Uyeda, E.R. Goldman, H. Mattoussi, Quantum dot bioconjugates for imaging, labelling and sensing, Nat. Mater. 4 (2005) 435-446.

[23] R. Gill, M. Zayats, I. Willner, Semiconductor quantum dots for bioanalysis, Angew. Chem., Int. Ed 47 (2008) 7602-7625.

[24] W.J. Parak, D. Gerion, D. Zanchet, A.S. Woerz, T. Pellegrino, C. Micheel, S.C. Williams, M. Seitz, R.E. Bruehl, Z. Bryant, C. Bustamante, C.R. Bertozzi, A.P. Alivisatos, Conjugation of DNA to silanized colloidal semiconductor nanocrystalline quantum dots, Chem. Mater. 14 (2002) 2113-2119.

[25] G.P. Mitchell, C.A. Mirkin, R.L. Letsinger, Programmed assembly of DNA functionalized quantum dots, J. Am. Chem. Soc. 121 (1999) 8122-8123.

[26] R. Gill, I. Willner, I. Shweky, U. Banin, Fluorescence resonance energy transfer in CdSe/ZnS-DNA conjugates: Probing hybridization and DNA cleavage, J. Phys. Chem. B 109 (2005) 23715-23719.

[27] D. Li, G. Li, W. Guo, P. Li, E. Wang, J. Wang, Glutathione-mediated release of functional plasmid DNA from positively charged quantum dots, Biomaterials 29 (2008) 2776-2782.

[28] G. Jiang, A.S. Susha, A.A. Lutich, F.D. Stefani, J. Feldmann, A.L. Rogach, Cascaded FRET in conjugated polymer/quantum dot/dye-labeled DNA complexes for DNA hybridization detection, ACS Nano 3 (2009) 4127-4131.

[29] T. Qiu, B. Zhang, Z.Y. Hu, J.H. Tang, H.P. Xie, B.R. Gu, Detection of DNA based on fluorescence resonance energy transfer of polyelectrolyte-protected CdTe quantum dots as energy donors, Analyst 137 (2012) 2608-2613.

[30] J. Lee, Y. Choi, J. Kim, E. Park, R. Song, Positively charged compact quantum dot-DNA complexes for detection of nucleic acids, Chemphyschem 10 (2009) 806-811.

[31] T. Qiu, D. Zhao, G. Zhou, Y. Liang, Z. He, Z. Liu, X. Peng, L. Zhou, A positively charged QDs-based FRET probe for micrococcal nuclease detection, Analyst 135 (2010) 2394-2399. 
[32] Y.C. Yeh, D. Patra, B. Yan, K. Saha, O.R. Miranda, C.K. Kim, V.M. Rotello, Synthesis of cationic quantum dots via a two-step ligand exchange process, Chem. Commun. 47 (2011) 30693071.

[33] N. Gaponik, D.V. Talapin, A.L. Rogach, K. Hoppe, E.V. Shevchenko, A. Kornowski, A. Eychmuller, $\mathrm{H}$. Weller, Thiol-capping of CdTe nanocrystals: An alternative to organometallic synthetic routes, J. Phys. Chem. B 106 (2002) 7177-7185.

[34] W.W. Yu, L.H. Qu, W.Z. Guo, X.G. Peng, Experimental determination of the extinction coefficient of CdTe, CdSe, and CdS nanocrystals, Chem. Mater. 15 (2003) 2854-2860.

[35] J. Moon, K.S. Choi, B. Kim, K.H. Yoon, T.Y. Seong, K. Woo, Aggregation-Free Process for Functional CdSe/CdS Core/Shell Quantum Dots, J. Phys. Chem. C 113 (2009) 7114-7119.

[36] J.B. Liu, X.H. Yang, K.M. Wang, X.X. He, Q. Wang, J. Huang, Y. Liu, Aggregation Control of Quantum Dots through Ion-Mediated Hydrogen Bonding Shielding, ACS Nano, 6 (2012) 4973-4983.

[37] J. R. Lakowicz, Principles of Fluorescence Spectroscopy; Kluwer Academic/Plenum Publishers: New York, 1999.

[38] A.L. Rogach, Fluorescence energy transfer in hybrid structures of semiconductor nanocrystals, Nano Today 6 (2011) 355-365.

[39] D. Yang, S. Xu, Q. Chen, Y. Wang, One system with two fluorescence resonance energy transfer (FRET) assembles among quantum dots, gold nanoparticles and enzyme, Colloids Surf. A 329 (2008) 38-43.

[40] S. Dhar, D.K. Rana, A. Sarkar, T.K. Mandal, S.C. Bhattacharya, Fluorescence resonance energy transfer from serum albumins to 1-anthracene sulphonate entrapped in reverse micellar nanocavities, Colloids Surf. A 369 (2010) 57-64.

[41] V. Biju, A. Anas, H. Akita, E. S. Shibu, T. Itoh, H. Harashima and M. Ishikawa, ACS Nano 6 (2012) 2012, 3776-3788. 

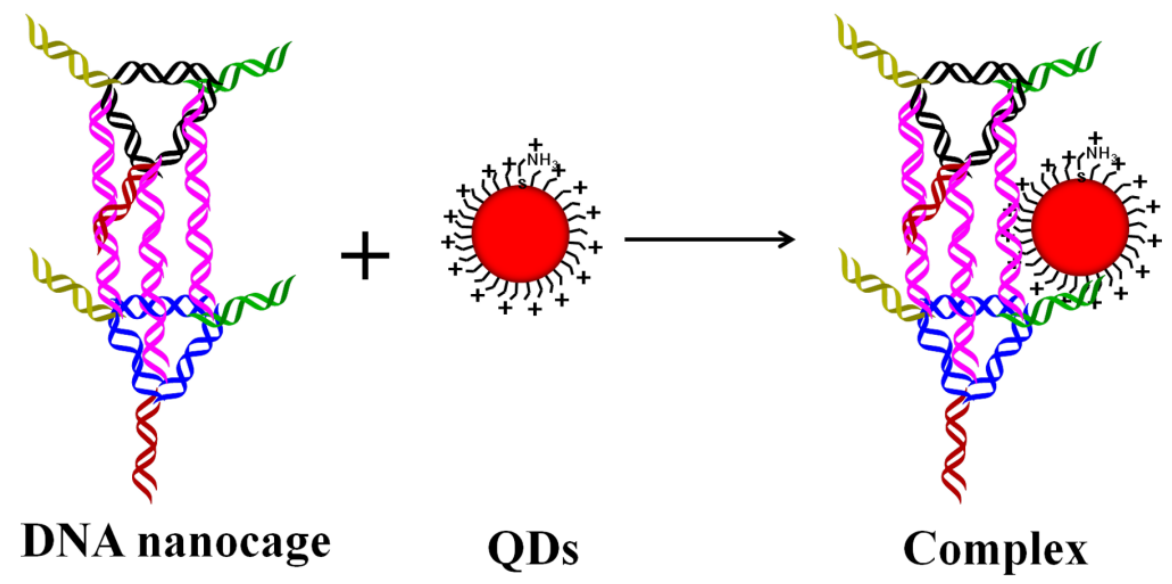

Complex 